\title{
The World at a Crossroads - The Need for Cartography and Cartographers: How cartographers can contribute as data integrators in finding solutions to global problems
}

\author{
Timothy Trainor \\ President, International Cartographic Association, Timothy Trainor - timtrainor4@gmail.com
}

Keywords: cartographers, data integration, maps

\begin{abstract}
:
The world is at a crossroads. The current global pandemic has had an impact on every person, their national and global economies, and their ability to preserve a sustainable environment. To understand the health impacts of such an intrusive consequence requires understanding of the pandemic's cause, its spread among populations, preventive measures to contain its spread and plans for protecting people from future outbreaks. Each one of these factors requires specific types of data and each data point requires location to make it meaningful. This is complicated because of the extent of the challenges. It is further complicated by the lack of timely data, the required location precision, and concerns over policy issues like privacy. Maps, mapping, and cartographers are needed as part of the formula for finding solutions on each of these factors.
\end{abstract}

The International Cartographic Association (ICA) is positioned to help in providing solutions. The structure of the ICA, through its various programs, is sufficiently flexible to respond to these critical issues in practical ways that yield useful results. As an example, new ICA Working Groups were recently established to focus on sustainability and bolstering national mapping and national geospatial organizations while a longer-term commitment centers on a revised research agenda to meet current and future cartographic and GIScience needs. These examples are in addition to other components of the ICA program including the ICA Commissions, conferences, and publications.

As the pandemic is global, local to global solutions are needed. The ICA has been actively engaged with the United Nations through the UN Committee of Experts on Global Geospatial Information Management. Three UN initiatives, two of which are geospatial frameworks, call out to the cartographic community for their help. The first is the Sustainable Development Goals that began in 2015 with a 2030 target to focus on a better understanding of national populations, their economies and their environments. Measuring and monitoring progress requires data, processes, systems, leadership, and commitment to be effective. The second effort is the Integrated Geospatial Information Framework, the next rendition of National Spatial Data Infrastructures (NSDI), that focuses on nine Strategic Pathways that are needed to create and maintain a sustainable geospatial program. The third initiative responds to the statistical and geospatial communities working together to create the Global Statistical Geospatial Framework which focuses on the integration of these two linked data types.

Cartographers can contribute in two areas. The first calls on their knowledge and expertise in working with different data types. For example, geospatially referenced statistical data oftentimes benefits from basic generalization principles such as combination, simplification, exaggeration and displacement. The absence of a small-area global geography sometimes inhibits the usefulness of statistical information which has become painfully evident during this pandemic. The second area that calls out to cartographers is to use their skills in making as many useful maps as possible. These maps need to show current local conditions, illuminate deficiencies, tell a story, and/or inform strategies and plans for addressing the many challenges we face between the pandemic and the underlying conditions of people, their communities, and circumstances oftentimes outside of their control.

The presentation shows examples of some of these points and the ICA's participation thus far. Opportunities for involvement are left to the imaginations of each of us. What is needed now is for cartographers and the cartographic community to act in ways that help in solving current challenges, informing some of the root causes and systemic problems that need attention while outlining approaches for a path forward in our ever increasingly complex world. 